\title{
Induced trees in graphs of large chromatic number
}

\author{
A.D. Scott \\ Department of Pure Mathematics \\ and Mathematical Statistics, \\ University of Cambridge, \\ 16 Mill Lane, Cambridge, CB2 1SB, England.
}

\begin{abstract}
Gyárfás and Sumner independently conjectured that for every tree $T$ and integer $k$ there is an integer $f(k, T)$ such that every graph $G$ with $\chi(G)>f(k, T)$ contains either $K_{k}$ or an induced copy of $T$. We prove a 'topological' version of the conjecture: for every tree $T$ and integer $k$ there is $g(k, T)$ such that every graph $G$ with $\chi(G)>g(k, T)$ contains either $K_{k}$ or an induced copy of a subdivision of $T$.
\end{abstract}




\section{$\S 1$ Introduction}

What can we say about the induced subgraphs of a graph $G$ with large chromatic number? Of course, one way for a graph to have large chromatic number is if it contains a large complete subgraph. However, if we consider graphs with large chromatic number and small clique number then we can ask what other subgraphs must occur. We can avoid any graph $H$ that contains a cycle since, as Erdös and Hajnal ([3], [1], [2]) showed, there are graphs with arbitrarily high girth and chromatic number; but what can we say about trees? Gyárfás [5] and Sumner [17] independently made the following beautiful and difficult conjecture.

Conjecture A. For every integer $k$ and tree $T$ there is an integer $f(k, T)$ such that every graph $G$ with

$$
\operatorname{cl}(G) \leq k
$$

and

$$
\chi(G) \geq f(k, T)
$$

contains an induced copy of $T$.

Let us rephrase this, using the notation of Gyárfás [6]. We call a class $\mathcal{G}$ of graphs $\chi$-bounded if there is a function $f$ such that $\chi(G) \leq f(\operatorname{cl}(G))$ for every $G \in \mathcal{G}$; we call $f$ a $\chi$-binding function. For instance, the class of perfect graphs is $\chi$-bounded with $f(k)=k$ as a $\chi$-binding function.

For a graph $H$, we write $\operatorname{Forb}(H)$ for the class of graphs that do not contain $H$ as an induced subgraph. For a family of graphs $\mathcal{H}$, we write $\operatorname{Forb}(\mathcal{H})$ for the class of graphs that contain no member of $\mathcal{H}$ as an induced subgraph. As we have remarked, $\operatorname{Forb}(H)$ is not $\chi$-bounded when $H$ contains a cycle. The conjecture of Gyárfás and Sumner asserts that $\operatorname{Forb}(T)$ is $\chi$-bounded for every tree $T$. In fact, an easy argument shows that the conjecture is equivalent to the assertion that $\operatorname{Forb}(H)$ is $\chi$-bounded iff $H$ is a forest.

If we do not demand that $T$ be induced, then the problem becomes much easier. Indeed, both Gyárfás, Szemerédi and Tuza [8] and Sumner [17] showed that if 
$\chi(G)=|T|=t$ and $T$ is coloured with $1, \ldots, t$, then any proper $t$-colouring of $G$ contains a subgraph isomorphic to $T$ and has the same labels.

It is also known that graphs with low clique number contain large induced trees. Erdős, Saks and Sós [4] proved that, for $k \geq 3$ and $n \geq 4$, every connected graph $G$ of order $n$ such that $\operatorname{cl}(G) \leq k$ contains an induced tree of order at least

$$
\frac{2 \log n}{(k-2) \log \log n}-3
$$

However, little can be said about the structure of such a tree.

Recently, attention has been given to the on-line version of the conjecture. Gyárfás and Lehel [7] proved that $\operatorname{Forb}\left(P_{5}\right)$ is on-line $\chi$-bounded and noted that $\operatorname{Forb}\left(P_{6}\right)$ is not on-line $\chi$-bounded; Kierstead, Penrice and Trotter [13] gave a better binding function and further results. Finally, Kierstead, Penrice and Trotter [14] proved the difficult result that, for any tree $T, \operatorname{Forb}(T)$ is on-line $\chi$-bounded iff $T$ has radius at most two. For a survey of these results, see Kierstead [9].

Returning to the conjecture, it follows easily from Ramsey's Theorem that $\operatorname{Forb}\left(K_{1, n}\right)$ is $\chi$-bounded for every $n$. (Indeed, suppose $\chi(G)>R(n, k)$ and $\operatorname{cl}(G) \leq k$. Then $G$ contains a vertex $x$ of degree at least $R(n, k)$, and so $\Gamma(x)$ must contain an independent set $S$ of size at least $n$, since $\operatorname{cl}(G) \leq k$; then $\{x\} \cup S$ induces $K_{1, n}$.) Gyárfás [6] showed that $\operatorname{Forb}\left(P_{n}\right)$ is $\chi$-bounded for every path $P_{n}$, and Hajnal and Rödl (see [12], [15], [16]) proved that $\operatorname{Forb}\left(T, K_{n, n}\right)$ is $\chi$-bounded for every tree $T$ and integer $n$. Significant progress was made by Gyárfás, Szemerédi and Tuza [8], who proved a special case of the conjecture for trees of radius two: for every tree $T$ of radius two there is a constant $c(T)$ such that every trianglefree graph $G$ such that $\chi(G) \geq c(T)$ contains an induced copy of $T$. Kierstead and Penrice [12] succeeded in generalizing this argument to prove the following.

Theorem B. Forb $(T)$ is $\chi$-bounded for every tree $T$ of radius two.

Very little else is known, however (though some special cases of the conjecture have been proved by Kierstead and Penrice [11] and Kierstead [10]; these results are both special cases of Corollary 2 below), and it seems that the full conjecture is rather difficult. Even partial results are therefore of interest. For instance, Sauer 
[16] notes that the conjecture is not even known to hold for subdivisions of stars; this will follow as a special case of Theorem 1 below.

For a graph $H$, let us write $\operatorname{Forb}^{*}(H)$ for the class of graphs that contain no subdivision of $H$ as an induced subgraph. (For instance, Forb* $\left(C_{3}\right)$ is the class of forests.) The main result of this paper is the following.

Theorem 1. Forb* $(T)$ is $\chi$-bounded for every tree $T$.

Equivalently, for every tree $T$ and positive integer $k$, every graph with sufficiently large chromatic number contains either $K_{k}$ or an induced copy of a subdivision of $T$.

This can be seen as a 'topological' version of the Gyárfás-Sumner conjecture, and allows us to demand trees with more structure than was previously possible (stars, paths, trees of radius two and a few other trees).

Consider now a tree $T$ that is a subdivision of a star (equivalently, $T$ contains at most one vertex of degree greater than two): whenever we subdivide $T$, we get a tree that contains $T$ as an induced subgraph. (In fact, subdivisions of stars are the only connected graphs with this property.) We therefore get the following result as an immediate corollary of Theorem 1.

Corollary 2. Let $T$ be a subdivision of a star. Then $\operatorname{Forb}(T)$ is $\chi$-bounded.

This answers the question mentioned by Sauer (and solves Problem 2.13 from [6]).

In order to prove Theorem 1, we will in fact prove a rather stronger result, which gives a bound on the extent to which our induced copy of $T$ is subdivided.

Theorem 3. For every tree $T$ there is an integer $t(T)$ such that the following assertion holds. For every integer $k$ there is an integer $c(k, T)$ such that every graph $G$ with

$$
\chi(G) \geq c(k, T)
$$


either contains $K_{k}$ or contains a subdivision of $T$ as an induced subgraph, with each edge of $T$ subdivided at most $t(T)$ times.

Now for a fixed tree $T$, there are only finitely many subdivisions of $T$ such that each edge of $T$ is subdivided at most $t(T)$ times. Theorem 3 can therefore be reformulated as follows.

Corollary 4. For every tree $T$ there is a finite family $T_{1}, \ldots, T_{s}$ of subdivisions of $T$ such that

$$
\bigcap_{i=1}^{s} \operatorname{Forb}\left(T_{i}\right)
$$

is $\chi$-bounded.

In $\S 2$ we give a proof of the main result. After a technical lemma, the proof is divided into two sections, depending on whether or not the 'local' chromatic number of our graph $G$ is large.

In $\S 3$ we make some remarks and suggest possible further applications of our method.

We use standard notation. For a graph $G$ and vertices $v, w \in V(G)$, we write $d_{G}(v, w)$ for the distance between $v$ and $w$, i.e. the length of a shortest path between them $\left(d_{G}(v, w)=\infty\right.$ if there is no such path). For $v \in V(G)$ and a positive integer $d$, we define

$$
B_{G}(v, d)=\left\{x \in V(G): d_{G}(v, x) \leq d\right\}
$$

and

$$
S_{G}(v, d)=\left\{x \in V(G): d_{G}(v, x)=d\right\} .
$$

If there is no ambiguity we write $d(v, w)$ for $d_{G}(v, w)$, etc.

For positive integers $a$ and $b$, let $T_{a}^{b}$ be the rooted tree of radius $b$ in which the root has degree $a$, every endvertex is distance $b$ from the root and every vertex that is not the root or an endvertex has degree $a+1$. Thus $T_{m}^{1} \cong K_{1, m}$. 


\section{$\S 2$. The main result}

In this section we prove Theorem 3. Our proof proceeds in several stages. We begin with a technical lemma about subdivisions, which will be used several times in the proof.

Lemma 5. For every triple of integers $m, d, k$ there is an integer $M(m, d, k)$ such that the following is true. Let $G$ be a graph with $\operatorname{cl}(G) \leq k$. Let $x_{1}, \ldots, x_{M}$ and $y_{1}, \ldots, y_{M}$ be vertices in $G$ such that, for $i=1, \ldots, M$,

$$
\Gamma\left(y_{i}\right)=\left\{x_{i}\right\}
$$

and

$$
d_{G}\left(x_{1}, x_{i}\right) \leq d .
$$

Then $G$ contains an induced subdivision of the star $K_{1, m}$ with endvertices $y_{1}$ and $m-1$ vertices from $y_{2}, \ldots, y_{M}$ such that each edge of $K_{1, m}$ is subdivided at most $d$ times.

Proof. We proceed by induction on $d$. If $d=1$, then $x_{1}$ is joined to $x_{2}, \ldots, x_{M}$. If $M \geq R(k, m-1)+1$ then since $\operatorname{cl}(G) \leq k$, there must be an independent set of size $m-1$ among $x_{2}, \ldots, x_{M}$, say $\left\{x_{2}, \ldots, x_{m}\right\}$. Then $G\left[\left\{x_{1}, \ldots, x_{m}\right\}\right]$ is a star $K_{1, m-1}$ with centre $x_{1}$, and $G\left[\left\{x_{1}, \ldots, x_{m}, y_{1}, \ldots, y_{m}\right\}\right]$ is a subdivision of $K_{1, m}$ with centre $x_{1}$ and each edge subdivided once.

Now suppose $d>1$ and the lemma is true for smaller values of $d$ (and any $m$, $k$ ). Let $x_{1}, \ldots, x_{M}, y_{1}, \ldots, y_{M}$ be as in the statement of the lemma. If we have $d_{G}\left(x_{1}, x_{i}\right) \leq d-1$ for at least $M(m, d-1, k)$ values of $i$ (from $\left.2, \ldots, M\right)$, then by the inductive hypothesis we can find the required subdivision of $K_{1, m}$. Thus we may assume that we have at least

$$
M_{0}=M-M(m, d-1, k)
$$

vertices $x_{i}$ with $d_{G}\left(x_{1}, x_{i}\right)=d$, say $x_{2}, \ldots, x_{M_{0}+1}$. For $i=2, \ldots, M_{0}+1$, let $P_{i}$ be a path of length $d$ from $x_{1}$ to $x_{i}$. Let

$$
S=\bigcup_{i=2}^{M_{0}+1} V\left(P_{i}\right)
$$


and, for $i=0, \ldots, d$, let

$$
S_{i}=\left\{x \in S: d_{G}\left(x_{1}, x\right)=i\right\} .
$$

Thus $S_{0}=\left\{x_{1}\right\}$ and $S_{d}=\left\{x_{2}, \ldots, x_{M_{0}+1}\right\}$. Now consider $G[S]$. If any $x \in S_{d-1}$ has $R(k, m-1)$ neighbours in $S_{d}$ then we are done: since $\operatorname{cl}(G) \leq k$, there must be an independent set of size $m-1$ in $\Gamma(x) \cap S_{d}$, say $\left\{x_{2}, \ldots, x_{m}\right\}$. Let $P$ be a path of length $d-1$ from $x_{1}$ to $x$. Then

$$
V(P) \cup\left\{x_{2}, \ldots, x_{m}\right\} \cup\left\{y_{1}, \ldots, y_{m}\right\}
$$

induces a subdivision of $K_{1, m}$ with centre $x$, endvertices $y_{1}, \ldots, y_{m}$, and each edge subdivided at most $d$ times.

Otherwise, we have

$$
\left|\Gamma(x) \cap S_{d}\right|<R(k, m-1)
$$

for every $x \in S_{d-1}$. Let $Z=\left\{z_{2}, \ldots, z_{M_{1}}\right\} \subset S_{d-1}$ be a minimal set such that every $x \in S_{d}$ has a neighbour in $Z$. It is clear from (2) that

$$
|Z| \geq\left|S_{d}\right| / R(k, m-1) .
$$

Furthermore, for every $z_{i} \in Z$ we can find $x_{z_{i}} \in S_{d}$ such that

$$
\Gamma\left(x_{z_{i}}\right) \cap Z=\left\{z_{i}\right\},
$$

or else we could replace $Z$ by $Z \backslash\left\{z_{i}\right\}$. Renumbering if necessary, we may assume that $x_{z_{i}}=x_{i}$ for each $i$.

We now find a large independent set among $x_{2}, \ldots, x_{M_{1}}$. Indeed, if

$$
|Z| \geq R(k+1, M(m, d-1, k)),
$$

then $x_{2}, \ldots, x_{M_{1}}$ contains an independent set of size $r=M(m, d-1, k)-1$, say $x_{2}, \ldots, x_{r}$. Consider the subgraph $H$ of $G$ induced by

$$
\left\{x_{1}, \ldots, x_{r}\right\} \cup\left\{z_{2}, \ldots, z_{r}\right\} \cup\left\{y_{1}\right\} \cup \bigcup_{i=1}^{d-2} S_{i} .
$$


We have $d_{H}\left(x_{1}, z_{i}\right)=d-1$ and $\Gamma_{H}\left(x_{i}\right)=\left\{z_{i}\right\}$, for $i=2, \ldots, r$, and $\Gamma_{H}\left(y_{1}\right)=\left\{x_{1}\right\}$. By the inductive hypothesis, $H$ contains an induced subdivision of $K_{1, m}$ with endvertices $y_{1}$ and $m-1$ vertices from $\left\{x_{2}, \ldots, x_{s}\right\}$, say $x_{2}, \ldots, x_{m}$, where each edge of $K_{1, m}$ is subdivided at most $d-1$ times. Adding $y_{2}, \ldots, y_{m}$, we get an induced subdivision of $K_{1, m}$ with endvertices $y_{1}, \ldots, y_{m}$ and each edge subdivided at most $d$ times.

From (1), (3) and (4), we deduce that

$$
M(m, d, k) \leq R(k+1, M(m, d-1, k)) \cdot R(k, m-1)+M(m, d-1, k) .
$$

We now turn to proving the main theorem. The proof is split into two lemmas: in the first we consider graphs that have chromatic number much larger than their 'local' chromatic number; in the second we consider graphs with large 'local' chromatic number. The main result will then follow by an easy argument.

Let us define a little notation. For any integer $r$ and graph $G$, we define the $r$-local chromatic number of $G$ to be

$$
\chi^{(r)}(G)=\max _{v \in V(G)} \chi(G[B(v, d)]) .
$$

Clearly $\chi^{(0)}(G)=1$, and $\chi^{(r)}(G)=\chi(G)$ whenever $r \geq \operatorname{diam}(G)$.

We prove a lemma about graphs $G$ for which $\chi^{(r)}(G)$ is much smaller than $\chi(G)$, for suitable $r$. In essence, the lemma states that for any tree $T$, every graph with small clique number, small local chromatic number and sufficiently large chromatic number contains a subdivision of $T$.

Lemma 6. For every tree $T$ and integer $k$ there exists a function $g: \mathbb{N} \rightarrow \mathbb{N}$ and integers $d, t$ such that for every integer $c$, every graph $G$ satisfying

$$
\begin{aligned}
\chi^{(d)}(G) & \leq c \\
\operatorname{cl}(G) & \leq k
\end{aligned}
$$


and

$$
\chi(G) \geq g(c)
$$

contains an induced subdivision $T^{*}$ of $T$, in which every edge is subdivided at most $t$ times.

Proof. It is enough to prove the theorem for trees of form $T_{a}^{b}$, since every tree $T$ is contained in $T_{s}^{s}$ for $s$ sufficiently large. Note that $T_{a}^{b}$ can be decomposed into $a$ copies of $T_{a}^{b-1}$, say $T_{1}, \ldots, T_{a}$, and an additional vertex $x$, where $x$ is joined to the root of $T_{i}$, for $i=1, \ldots, a$.

The idea of the proof is simple: arguing by induction on $b=\operatorname{rad}\left(T_{a}^{b}\right)$, we take induced copies of a tree containing $T_{a}^{b-1}$ and join $a$ of them together to get $T_{a}^{b}$. However, there are a couple of technical difficulties: a vertex in one copy of $T_{a}^{b-1}$ may be adjacent to vertices in another copy; and a vertex adjacent to one vertex in a given copy of $T_{a}^{b-1}$ may be adjacent to other vertices in that copy as well. Thus we demand that our copies of $T_{a}^{b-1}$ are not too close together, and that each copy is 'spread out' in $G$, in the following sense.

We say that an induced subgraph $T^{*}$ of $G$ is a $\left(T_{a}^{b}, t\right)$-structure in $G$ if the following two conditions are satisfied.

1. $T^{*}$ is a subdivision of $T_{a}^{b}$ such that each edge is subdivided at most $t$ times.

2. Let $x^{*}$ be the centre of $T^{*}$, and let $T_{1}^{*}, \ldots, T_{a}^{*}$ be the induced subdivisions of $T_{a}^{b-1}$ corresponding to $T_{1}, \ldots, T_{a}$ in the decomposition of $T_{a}^{b}$ given above. Then, for $1 \leq i \leq a$,

$$
d_{G}\left(x, T_{i}^{*}\right) \geq 3
$$

and, for $1 \leq i<j \leq a$,

$$
d_{G}\left(T_{i}^{*}, T_{j}^{*}\right) \geq 3
$$

Now fix $k$. We prove by induction on $b$ that for every pair of integers $a$ and $b$ there exists a function $g_{a, b}: \mathbb{N} \rightarrow \mathbb{N}$ and integers $d, t$ such that, for every integer $c$, whenever $G$ is a graph such that

$$
\chi^{(d)}(G) \leq c
$$


and

$$
\operatorname{cl}(G) \leq k,
$$

and $X \subset V(G)$ satisfies

$$
\chi(G[X]) \geq g_{a, b}(c),
$$

then we can find an induced $\left(T_{a}^{b}, t\right)$-structure with all its endvertices in $X$. Note that this is stronger than demanding an induced $\left(T_{a}^{b}, t\right)$-structure in $G[X]$, since we may have $d_{G[X]}(x, y)<d_{G}(x, y)$ for vertices $x, y \in X$.

For $b=0$ the assertion is trivial. Suppose that $b>0$ and that the assertion is true for smaller values of $b$. By the inductive hypothesis, we may pick constants $d, t$ and a function $g: \mathbb{N} \rightarrow \mathbb{N}$ such that, for every integer $c$, whenever $G$ is a graph such that

$$
\chi^{(d)}(G) \leq c
$$

and

$$
\operatorname{cl}(G) \leq k
$$

and $X \subset V(G)$ satisfies

$$
\chi(G[X]) \geq g(c)
$$

then we can find a $\left(T_{2 a}^{b-1}, t\right)$-structure in $G$, with all its endvertices in $X$. Increasing $t$ if necessary, we may assume $t \geq d$. Let us note that any $\left(T_{2 a}^{b-1}, t\right)$-structure has radius at most

$$
D=(t+1)(b-1) .
$$

We show that, for every integer $c$, if

$$
\chi^{(3 D+10)}(G) \leq c
$$

and

$$
\operatorname{cl}(G) \leq k,
$$

and $X \subset V(G)$ satisfies

$$
\chi(G[X]) \geq M(c+g(c))
$$

for sufficiently large $M$ (depending on $T$ and $k$ ), then $G$ contains a $\left(T_{a}^{b}, 14 D+42\right)$ structure. 
Suppose that $G$ and $X$ satisfy (8) and (9). Let $T_{1}, \ldots, T_{p}$ be a maximal set of $\left(T_{2 a}^{b-1}, t\right)$-structures in $G$ with centres, say, $x_{1}, \ldots x_{p}$, such that each $T_{i}$ has all endvertices in $X$ and, for $i \neq j$,

$$
d\left(x_{i}, x_{j}\right) \geq 2 D+10
$$

Note that $V\left(T_{i}\right) \subset B\left(x_{i}, D\right)$ for each $i$, so for $i \neq j$ we have

$$
d\left(T_{i}, T_{j}\right) \geq 10
$$

Now consider

$$
W=\bigcup_{i=1}^{p} B\left(x_{i}, 3 D+10\right)
$$

There are no $\left(T_{2 a}^{b-1}, t\right)$-structures in $G$ with endvertices in $X \backslash W$, or else $T_{1}, \ldots, T_{p}$ would not be maximal. (If $T_{p+1}$ were another such $\left(T_{2 a}^{b-1}, t\right)$-structure, with centre $x_{p+1}$, say, then since all endvertices of $T_{p+1}$ are contained in $X \backslash W$ and the radius of $T_{p+1}$ is at most $D$, we would have $d\left(x_{p+1}, X \backslash W\right) \leq D$. Therefore $d\left(x_{i}, x_{p+1}\right) \geq d\left(x_{i}, X \backslash W\right)-d\left(x_{p+1}, X \backslash W\right) \geq 2 D+10$, and so we could take $T_{1}, \ldots, T_{p+1}$ instead of $T_{1}, \ldots, T_{p}$.) Thus, by the inductive hypothesis, we must have

$$
\chi(G[X \backslash W])<g(c)
$$

and so

$$
\begin{aligned}
\chi(G[W]) & \geq \chi(G[X])-\chi(G[X \backslash W]) \\
& \geq(M-1)(c+g(c)) .
\end{aligned}
$$

We now try to join some of the $\left(T_{2 a}^{b-1}, t\right)$-structures $T_{1}, \ldots, T_{p}$ together to get a $\left(T_{a}^{b}, 14 D+42\right)$-structure. We begin by showing that some $x_{i}$ is not too far from $M$ other vertices amongst $x_{1}, \ldots, x_{p}$. Let $\mu$ be the following colouring of $W$ : for each $x \in W$ let

$$
j(x)=\min _{i=1, \ldots, p}\left\{d\left(x, x_{i}\right)\right\}
$$

and define

$$
\mu(x)=\min \left\{i: d\left(x, x_{i}\right)=j(x)\right\}
$$


(Note that it follows from (11) that $j(x)$ and $\mu(x)$ do not depend on whether we take the distance in $G$ or the distance in $G[W]$.) Let the $\mu$-colour classes be $C_{1}, \ldots, C_{p}$. It is easily checked that $G\left[C_{i}\right]$ is connected for each $i$ : indeed, if $x \in C_{i}$ and $P$ is a path of length $d\left(x, x_{i}\right)$ from $x_{i}$ to $x$ then $V(P) \subset C_{i}$. Now from (8) and (11) we have

$$
\chi\left(G\left[C_{i}\right]\right) \leq c
$$

for $i=1, \ldots, p$, since $C_{i} \subset B\left(x_{i}, 3 D+10\right)$. Let $\lambda_{i}: C_{i} \rightarrow[c]$ be a colouring of $G\left[C_{i}\right]$, for $i=1, \ldots, p$, and for $x \in W$ define

$$
\lambda(x)=\lambda_{\mu(x)}(x) .
$$

Thus adjacent vertices in $W$ have the same $\lambda$-colour only if they are in different $\mu$-colour classes.

Now consider the graph $H$ with vertices $1, \ldots, p$ and an edge between $i$ and $j$ iff

$$
e\left(C_{i}, C_{j}\right)>0
$$

If $\chi(H)<M$, then let $\nu$ be a colouring of $H$ with $M-1$ colours. We get a proper colouring of $X$ by colouring each $x \in X$ with the ordered pair

$$
\langle\lambda(x), \nu(\mu(x))\rangle .
$$

Thus $\chi(G[W]) \leq c(M-1)$, which contradicts (12). Therefore, $\chi(H) \geq M$ and so some $C_{i}$ satisfies (13) for at least $M-1$ values of $j$.

Let us suppose

$$
e\left(C_{1}, C_{j}\right)>0
$$

for $j=2, \ldots, M$.

The idea now is to take the $\left(T_{2 a}^{b-1}, t\right)$-structures $T_{2}, \ldots, T_{M}$ in $C_{2}, \ldots, C_{M}$ and connect them together through $C_{1}$. We know that $G\left[C_{i}\right]$ is connected for each $i$; it follows from (14) that $G\left[C_{1} \cup C_{i}\right]$ is connected for $i=2, \ldots, M$. It also follows from (10) and the definition of the $C_{i}$ that there are no edges between $C_{1}$ and $B\left(x_{i}, D+3\right)$. Let $P_{i}$ be a shortest path in $G\left[C_{1} \cup C_{i}\right]$ from $x_{1}$ to $B\left(x_{i}, D+2\right)$; by (11) we have

$$
\left|P_{i}\right| \leq 5 D+19
$$


Suppose

$$
P_{i}=x_{1} \ldots w_{i} v_{i}
$$

where $d\left(w_{i}, x_{i}\right)=D+3$, and $d\left(v_{i}, x_{i}\right)=D+2$. Let

$$
S=\bigcup_{i=2}^{M} V\left(P_{i}\right)
$$

Now it is clear from (10) and the definition of the $C_{i}$ that $\Gamma\left(v_{i}\right) \cap S=\left\{w_{i}\right\}$ for $i=2, \ldots, M$, and $d\left(v_{i}, v_{j}\right) \geq 6$ for $i \neq j$. It follows from (15) and (16) that $d_{G[S]}\left(x_{1}, v_{j}\right) \leq 5 D+19$. Applying Lemma 5 to $G[S]$ (with an extra pendant vertex attached to $x_{1}$ ), we see that if

$$
M>M(a+2,5 D+19, k)
$$

then $G[S]$ contains an induced subdivision of the star $K_{1, a+2}$ with endvertices from $w, v_{2}, \ldots, v_{M}$, and thus an induced subdivision $U$ of $K_{1, a+1}$ with endvertices from $v_{2}, \ldots, v_{M}$, where each edge is subdivided at most $5 D+19$ times. Let the centre of $U$ be $v$; we may assume that $U$ has endvertices $v_{2}, \ldots, v_{a+2}$. Then $d\left(v, v_{i}\right)<3$ for at most one $i$ (since $d\left(v_{i}, v_{j}\right) \geq 6$ for $i \neq j$ ), so we may assume

$$
d\left(v, v_{i}\right) \geq 3
$$

for $i=2, \ldots, a+1$.

$U$ will form the centre of our induced subdivision of $T_{a}^{b}$. Our aim now is to join $U$ to $T_{2}, \ldots, T_{a+1}$. Recall that, by definition, $d\left(v_{i}, x_{i}\right)=D+2$ and $d\left(w_{i}, x_{i}\right)=D+3$ Let $Q_{i}$ be a shortest path of the form $w_{i} v_{i} \cdots y_{i} t_{i}$, where every vertex after $v_{i}$ belongs to $B\left(x_{i}, D+1\right)$ and $t_{i} \in V\left(T_{i}\right)$.

Now, since $T_{i}$ is a $\left(T_{2 a}^{b-1}, t\right)$-structure, it has subtrees $T_{1}^{*}, \ldots, T_{2 a}^{*}$, where $T_{j}^{*}$ is joined to $x_{i}$ by a path $R_{j}=x_{i} \cdots x_{l}^{*}$ of length at least three. Let $U_{1}, \ldots, U_{2 a}$ be the components of $T_{i} \backslash\left\{x_{i}\right\}$, where $V\left(T_{j}^{*}\right) \subset V\left(U_{j}\right)$, for each $j$. We construct an induced subdivision of $T_{a}^{b-1}$ with its root joined by a path to $y_{i}$. If $y_{i}$ has neighbours in at most one of the $U_{j}$, say in $U_{s}$, then delete $U_{s}$ and join $y_{i}$ to $x_{i}$ by a shortest path $P$ in $G\left[\left\{x_{i}, y_{i}\right\} \cup V\left(U_{s}\right)\right]$; our subdivision is induced by $V(P)$ and any $b-1$ sets from $\left\{V\left(U_{j}\right): j \neq s\right\}$. Otherwise, $y_{i}$ has neighbours in more than 
one $U_{i}$. In this case, it follows from (6) that $y_{i}$ can have neighbours in at most one $T_{i}^{*}$; we may suppose $y_{i}$ has no neighbours in $T_{1}^{*}, \ldots, T_{2 a-1}^{*}$. If $y$ has neighbours in at most $a-1$ of the $U_{i}$, say among $U_{1}, \ldots, U_{a-1}$, then join $y_{i}$ to $x_{i}$ by a shortest path $P$ in $G\left[\left\{x_{i}, y_{i}\right\} \cup \bigcup_{i=1}^{a-1} U_{i}\right]$; our subdivision is induced by $V(P) \cup \bigcup_{j=a}^{2 a-1} V\left(U_{j}\right)$. Otherwise, we may assume that $y_{i}$ has neighbours in $U_{1}, \ldots, U_{a}$. In this case, join $y_{i}$ to $T_{j}^{*}$ by a shortest path $S_{j}$ in $\left\{y_{i}\right\} \cup U_{j}$, and take $\bigcup_{j=1}^{a}\left(V\left(S_{j}\right) \cup V\left(T_{j}^{*}\right)\right)$. It is easily checked that for each $i$ we obtain an induced subdivision of $T_{a}^{b-1}$, joined to $U$ by a path; adding $U$, we obtain an induced subdivision of $T_{a}^{b}$. Furthermore, it follows from (7) and (15) that this induced subdivision is a $\left(T_{a}^{b}, 14 D+42\right)$ structure.

We have now dealt with graphs that have low 'local' chromatic number. How will a more general proof of Theorem 3 proceed? Well, our aim is to argue by induction on $|T|$. Suppose we have proved the theorem for smaller trees: let

$$
N=\max \{c(k, S): S \text { is a tree and }|S|<|T|\},
$$

where $c(k, S)$ is the minimum $c$ satisfying Theorem 3 . Let $g, k, d$ be as in Lemma 6 , and let $G$ be a graph with large chromatic number.

How can we find an induced subdivision of $T$ ? If we have some $X \subset V(G)$ such that $\chi(G[X])>g\left(\chi^{(d)}(G[X])\right)$ then we are done immediately by Lemma 6 . We are also done, by the inductive hypothesis, if $\chi(G[\Gamma(x)])>c(k-1, T)$.

What other structures guarantee an induced copy of $T$ ? Let us call a subset $X \subset V(G)$ well-covered in $G$ if for each $x \in X$ there exists $x^{\prime} \in V(G) \backslash X$ such that $\Gamma\left(x^{\prime}\right) \cap X=\{x\}$. If we can find a well-covered subset $X$ of $V(G)$ that induces a graph with chromatic number at least $N$, then $G[X]$ contains an induced copy of $T \backslash\{t\}$, where $t$ is an endvertex of $T$. However, since $X$ is well-covered, we can add a vertex from $V(G) \backslash X$ to get an induced copy of $T$.

What do we do if none of these structures can be found in $G$ ? The next lemma says that, provided that a ball around some vertex has high enough chromatic number, then we can build a tree from that vertex. 
Lemma 7. Let $T$ be a tree, let $g: \mathbb{N} \rightarrow \mathbb{N}$ be an unbounded increasing function and let $N, L, d$ be constants. Let $G$ be a graph such that

$$
\chi(G[B(x, 1)])<N
$$

for every $x \in G$, such that no well-covered subset $X \subset V(G)$ satisfies

$$
\chi(G[X])>L
$$

and such that whenever $H$ is an induced subgraph of $G$, we have

$$
\chi(H)<g\left(\chi^{(d)}(H)\right) .
$$

Then there exist constants $C(T, N, L, d)$ and $t(T, d)$ such that if, for some $x \in$ $V(G)$,

$$
\chi\left(G\left[B_{G}(x, d)\right]\right)>C,
$$

then there exists an induced subdivision of $T$, or $T$ with a pendant vertex, that contains $x$, and in which each edge is subdivided at most $t$ times.

Proof. We prove this for trees of form $T_{a}^{b}$ by induction on $b=\operatorname{rad}(T)$, with the additional condition that $x$ corresponds to the root of $T_{a}^{b}$ or else corresponds to a pendant vertex added to the root of $T_{a}^{b}$.

For $b=0$ the result is trivial. Suppose $b>0$, and we have proved the lemma for smaller values of $b$. As in Lemma 6 , we remark that $T$ can be decomposed into $a$ copies of $T_{a}^{b-1}$ with their centres joined to a central vertex $y$. The idea of the proof is to take copies of $T_{a}^{b-1}$ rooted in $S_{G}(x, i)$, for some $i<d$, and join them together in $B_{G}(x, i-1)$.

Let $C_{0}=C\left(T_{a}^{b-1}, N, L, d+1\right)$, and let $C$ be a large constant. Suppose

$$
\chi\left(G\left[B_{G}(x, d)\right]\right)>C,
$$

so for some $d_{0} \leq d$,

$$
\chi\left(G\left[S_{G}\left(x, d_{0}\right)\right]\right) \geq C / 2
$$


since

$$
\chi\left(G\left[B_{G}(x, d)\right]\right) \leq \max _{i=1, \ldots, d}\left(\chi\left(G\left[S_{G}(x, i)\right]\right)+\chi\left(G\left[S_{G}(x, i-1)\right]\right)\right) .
$$

Let $X=S_{G}\left(x, d_{0}\right)$, and let $T_{1} \subset S_{G}\left(x, d_{0}-1\right)$ be minimal such that

$$
\left|\Gamma(x) \cap T_{1}\right|>0
$$

for all $x \in X$. By minimality of $T_{1}$, for each $s \in T_{1}$ we can find $x_{s} \in X$ such that

$$
\Gamma\left(x_{s}\right) \cap T_{1}=\{s\}
$$

Define $U_{1}=\left\{x_{s}: s \in T_{1}\right\}$.

We define sets $T_{1}, \ldots, T_{p}$ and $U_{1}, \ldots, U_{p}$ as follows. Given the sets $T_{1}, \ldots, T_{j}$ and $U_{1}, \ldots, U_{j}$, if $\bigcup_{i=1}^{j} U_{i}=X$ then set $p=j$ and stop. Otherwise, let $X_{j}=X \backslash$ $\bigcup_{i=1}^{j} U_{i}$, and let $T_{j+1} \subset T_{j}$ be minimal such that $\left|\Gamma(x) \cap T_{j+1}\right|>0$ for all $x \in X_{j}$. As before, for each $y \in T_{j+1}$ we can find $x_{y} \in X_{j}$ such that $\Gamma\left(x_{y}\right) \cap T_{j+1}=\{y\}$.

Define

$$
U_{j+1}=\left\{x_{y}: y \in T_{j+1}\right\}
$$

Clearly, for each $j, U_{j}$ is well-covered in $G$ by $T_{j}$, so by (19) we have

$$
\chi\left(G\left[U_{j}\right]\right) \leq L
$$

Furthermore, since $T_{j} \supset T_{j+1} \supset \cdots$ it follows that every vertex $x \in U_{j}$ has at most one neighbour in $T_{i}$ for $i \geq j$. We know from (21) that

$$
\chi\left(G\left[\bigcup_{j=1}^{p} U_{j}\right]\right)=\chi(G[X]) \geq C / 2,
$$

and from (22) that, for each $l$,

$$
\begin{aligned}
\chi\left(G\left[\bigcup_{j=1}^{l+1} U_{j}\right]\right) & \leq \chi\left(G\left[\bigcup_{i=1}^{l} U_{j}\right]\right)+\chi\left(G\left[U_{l+1}\right]\right) \\
& \leq \chi\left(G\left[\bigcup_{j=1}^{l} U_{j}\right]\right)+L .
\end{aligned}
$$


Let $s$ be minimal such that

$$
\chi\left(G\left[\bigcup_{j=1}^{s} U_{j}\right]\right) \geq g\left(C_{0}\right)
$$

( $s$ is well-defined provided $C$ is sufficiently large) and let

$$
Y_{1}=\bigcup_{j=1}^{s} U_{j}
$$

Our aim now is to find (a subdivision of) an induced copy of $T_{a}^{b-1}$ with one vertex (its root, or a pendant vertex attached to its root) in $T_{1}$ and the remainder of its vertices in $Y_{1}$. Now from (22) and (23) it follows that

$$
g\left(C_{0}\right) \leq \chi\left(G\left[Y_{1}\right]\right) \leq g\left(C_{0}\right)+L
$$

By (20), we have $\chi\left(G\left[Y_{1}\right]\right)<g\left(\chi^{(d)}\left(G\left[Y_{1}\right]\right)\right)$, and so, for some $y \in Y_{1}$,

$$
\chi\left(B_{G\left[Y_{1}\right]}(y, d)\right)>C_{0} .
$$

Pick $z_{1} \in \Gamma(y) \cap T_{1}$, and consider $H=G\left[\left\{z_{1}\right\} \cup X\right]$. Since $B_{H}\left(z_{1}, d+1\right) \supset$ $B_{G\left[Y_{1}\right]}(y, d)$ we have $\chi\left(B_{H}\left(z_{1}, d+1\right)\right)>C_{0}$. Thus by our inductive hypothesis, we can find an induced subdivision of $T_{a}^{b-1}$ in $H$ such that $z_{1}$ corresponds to its root, or an induced subdivision of $T_{a}^{b-1}$ with a pendant vertex corresponding to $z_{1}$ added to its root, where each edge has been subdivided at most $t\left(T_{a}^{b-1}\right)$ times. Call this $H_{1}$. Note that $H_{1}$ has at most

$$
h=\left(\left|T_{a}^{b-1}\right|+1\right)\left(t\left(T_{a}^{b-1}\right)+1\right)
$$

vertices.

We want now to define further induced trees $H_{2}, H_{3}, \ldots$, with roots $z_{2}, z_{3}, \ldots$, such that there is no edge in $G$ between $H_{i} \backslash z_{i}$ and $H_{j} \backslash z_{j}$ for $i \neq j$. Thus we will have to avoid the vertices adjacent to $H_{1} \backslash z_{1}$. With this in mind, we define

$$
\begin{aligned}
S_{1} & =\left(V\left(H_{1}\right) \cup \bigcup_{x \in V\left(H_{1}\right)} \Gamma(x)\right) \cap X \\
S_{2} & =\left\{x \in T_{s+1}:\left|\Gamma(x) \cap V\left(H_{1}\right) \backslash\left\{z_{1}\right\}\right|>0\right\} \\
S_{3} & =\bigcup_{x \in S_{2}} \Gamma(x) \cap X .
\end{aligned}
$$


Clearly, by (18),

$$
\begin{aligned}
\chi\left(G\left[S_{1}\right]\right) & \leq \sum_{x \in V\left(H_{1}\right)} \chi(G[(\Gamma(x) \cap X) \cup\{x\}]) \\
& \leq \sum_{x \in V\left(H_{1}\right)} \chi(G[B(x, 1)]) \\
& \leq h N .
\end{aligned}
$$

Now $\left|S_{2}\right| \leq h$, since each $x \in V\left(H_{1}\right) \backslash\left\{z_{1}\right\}$ belongs to $U_{l}$ for some $l \leq s$, and so since (as remarked above) $T_{l} \supset T_{s+1}$ we have

$$
\left|\Gamma(x) \cap T_{s+1}\right| \leq\left|\Gamma(x) \cap T_{l}\right|=1 .
$$

Thus $\left|S_{2}\right| \leq\left|H_{1}\right|=h$, and so by (18) we have

$$
\begin{aligned}
\chi\left(G\left[S_{3}\right]\right) & \leq \sum_{x \in S_{2}} \chi(G[\Gamma(x) \cap X]) \\
& \leq h N .
\end{aligned}
$$

Now define

$$
X^{\prime}=X \backslash\left(Y_{1} \cup S_{1} \cup S_{3}\right)
$$

and let $T_{1}^{\prime} \subset T_{s+1} \backslash S_{2}$ be a minimal cover for $X^{\prime}$. We have by (24) that

$$
\begin{aligned}
\chi\left(G\left[X^{\prime}\right]\right) & \geq \frac{C}{2}-\chi\left(G\left[Y_{1}\right]\right)-\chi\left(G\left[S_{1}\right]\right)-\chi\left(G\left[S_{3}\right]\right) \\
& \geq \frac{C}{2}-g\left(C_{0}\right)-L-2 h N .
\end{aligned}
$$

Provided $C \geq 2 M(a, d+1, k)\left(g\left(C_{0}\right)+L+2 h N\right)$, we can repeat the process $M(a, d+$ $1, k)$ times, to get induced subdivisions $H_{1}, \ldots, H_{M(a, d+1, k)}$ of $T_{a+1}^{b-1}$ rooted at $z_{i} \in S(x, i-1)$, or with root joined to $z_{i}$ by a path of length at most $t\left(T_{a+1}^{b-1}\right)$, and all remaining vertices in $S(x, i)$. By the definition of (25), the only possible edge between $H_{i}$ and $H_{j}$, for $i \neq j$, is $z_{i} z_{j}$. Applying Lemma 5 to the graph formed by joining $x$ to $z_{1}, \ldots, z_{M(a, d+1, k)}$ by shortest paths (and adding a pendant vertex to the $z_{i}$ ), we get the required induced subdivision of $T_{a}^{b}$, or $T_{a}^{b}$ with a pendant vertex, with each edge subdivided at most

$$
2 t\left(T_{a+1}^{b-1}\right)+2 d
$$

times. 
Theorem 3 now follows easily by a double induction on $k$ and $T$. Indeed, for $k=1$ the result is immediate. Suppose $k>1$ and we know the result for smaller values of $k$ (for all $T$ ), and for $k$ and smaller trees. As remarked above, if

$$
\chi\left(G\left[B_{G}(x, 1)\right]\right)>c(k-1, T)
$$

for any $x \in V(G)$, or we have a well-covered subset $X \subset V(G)$ with

$$
\chi(G[X])>c(k, T \backslash\{x\})
$$

where $x$ is an endvertex of $T$, then we are done. By Lemma 6 , there are constants $t$ and $d$, and a function $g$, such that if

$$
\chi(G[X]) \geq g\left(\chi^{(d)}(G[X])\right)
$$

for any $X \subset V(G)$, then $G$ contains an induced subdivision of $T$ with each edge subdivided at most $t$ times. If $(26)$ is not satisfied for any $X \subset V(G)$, and $\chi(G)>g(C(T, N, L, d))$, then

$$
\chi\left(G\left[B_{G}(x, d)\right]\right)>C(T, N, L, d)
$$

for some $x \in V(G)$. But then Lemma 7 gives us the required induced subdivision of $T$.

\section{§3. Remarks}

We can actually strengthen Theorem 3 slightly, in that we can take $t$ to be dependent only on the radius of $T$. In other words, for every integer $r$ there is an integer $t(r)$ such that, for any tree $T$ of radius $r$ and any integer $k$, every graph with sufficiently large chromatic number contains a copy of $K_{k}$ or else an induced copy of $T$ in which each edge is subdivided at most $t$ times. This follows with fairly easy modifications to the proofs of Lemmas 5,6 and 7 .

The bounds for $t$ that follow from the proof above are rather large, and grow exponentially in $|T|$. It would be interesting to give a smaller bound. 
We believe that the methods we have developed here should have further application. Motivated by the Strong Perfect Graph Conjecture, Gyárfás [6] has made a number of conjectures about $\chi$-bounded families of graphs. For any integer $m$, let

$$
\mathcal{H}_{m}=\left\{C_{2 m+1}, C_{2 m+3}, \ldots\right\}
$$

and

$$
\mathcal{C}_{m}=\left\{C_{m}, C_{m+1}, \ldots\right\}
$$

Gyárfás conjectured that $\operatorname{Forb}\left(\mathcal{H}_{2}\right)$ is $\chi$-bounded and that $\operatorname{Forb}\left(\mathcal{C}_{m}\right)$ is $\chi$-bounded for $m \geq 4$. $\left(\operatorname{Forb}\left(\mathcal{C}_{4}\right)\right.$ is the family of triangulated graphs, which is known to be perfect.) He also made the stronger conjecture that $\operatorname{Forb}\left(\mathcal{H}_{m}\right)$ is $\chi$-bounded for $m \geq 2$. We have so far been able to prove only that $\operatorname{Forb}\left(\mathcal{H}_{2} \cup \mathcal{C}_{m}\right)$ is $\chi$-bounded for every integer $m$, but hope that our methods can be exploited for further questions of this type.

All the conjectures mentioned above ask whether, for some family $\mathcal{F}$ of graphs, $\operatorname{Forb}(\mathcal{F})$ is $\chi$-bounded; clearly, many similar questions can be asked. In particular, the case when $\mathcal{F}$ consists of the subdivisions of a single graph $H$, so that $\operatorname{Forb}(\mathcal{F})=$ Forb $^{*}(H)$, seems interesting: Theorem 1 deals with the case when $H$ is a tree, and one of the conjectures of Gyárfás concerns $\operatorname{Forb}\left(\mathcal{C}_{m}\right)=\operatorname{Forb}^{*}\left(C_{m}\right)$. We make the following stronger conjecture.

Conjecture 8. Forb* $(H)$ is $\chi$-bounded for every graph $H$.

\section{References}

[1] P. Erdős, Graph Theory and Probability, Canadian J. Math 11 (1951), 34-38.

[2] P. Erdős, Graph Theory and Probability II, Canadian J. Math 11 (1961), 346-352.

[3] P. Erdös and A. Hajnal, On chromatic number of graphs and set systems, Acta Math. Sci. Hung. 17 (1966), 61-69.

[4] P. Erdös, M. Saks and V.T. Sós, Maximum induced trees in graphs,, J. Comb. Theory Ser. B 41 (1986), 61-79. 
[5] A. Gyárfás, On Ramsey covering-numbers, Coll. Math. Soc. János Bolyai, in Infinite and Finite Sets, North Holland/American Elsevier, New York (1975), 10.

[6] A. Gyárfás, Problems from the world surrounding perfect graphs, Zastowania Matematyki Applicationes Mathematicae XIX (1985), 413-441.

[7] A. Gyárfás and J. Lehel, Effective On-line Colouring of $P_{5}$-free graphs, Combinatorica 11 (1991), 181-184.

[8] A. Gyárfás, E. Szemerédi and Zs. Tuza, Induced subtrees in graphs of large chromatic number, Discrete Math. 30 (1980), 235-344.

[9] H.A. Kierstead, Recursive and On-line Graph Coloring, to appear.

[10] H.A. Kierstead, Long stars specify $\chi$-bounded classes, in Coll Math Soc Janós Bolyai 60: Sets, Graphs and Numbers, Budapest (1991), 421-428.

[11] H.A. Kierstead and S.G. Penrice, Recent results on a conjecture of Gyárfás, Congressus Numerantium $\mathbf{7 9}$ (1990), 182-186.

[12] H.A. Kierstead and S.G. Penrice, Radius two trees specify $\chi$-bounded classes, $J$. Graph Theory 18 (1994), 119-129.

[13] H.A. Kierstead, S.G. Penrice and W.T. Trotter, On-line and first-fit coloring of graphs which do not induce $P_{5}$, SIAM J. Disc. Math. 8 (1995), 485-498.

[14] H.A. Kierstead, S.G. Penrice and W.T. Trotter, On-line colouring and recursive graph theory, SIAM J. Disc. Math. 7 (1994), 72-89.

[15] H.A. Kierstead and V. Rödl, Applications of hypergraph colouring to colouring graphs that do not induce certain trees, Discrete Math. 150 (1996), 187-193.

[16] N. Sauer, Vertex partition problems, in Combinatorics, Paul Erdős is Eighty, Bolyai Society Mathematical Studies, ed. D. Miklós, V.T. Sós and T. Szőnyi (1993), 361-377.

[17] D.P. Sumner, Subtrees of a graph and chromatic number, in The Theory and Applications of Graphs, ed. G. Chartrand, John Wiley \& Sons, New York (1981), 557-576. 